\title{
Dispersal Distances for Airborne Spores Based on Deposition Rates and Stochastic Modeling
}

\author{
Anders Stockmarr, Viggo Andreasen, and Hanne Østergård
}

First and third authors: Biosystems Department, Ris $\varnothing$ National Laboratory, Technical University of Denmark, P.O. Box 49, DK-4000 Roskilde, Denmark; first author: National Veterinary Institute, Technical University of Denmark, Bülowsvej 27, DK-1790 Frederiksberg C, Denmark; and second author: IMFUFA, Department of Sciences, Roskilde University, DK-4000 Roskilde, Denmark. Accepted for publication 9 April 2007.

\begin{abstract}
Stockmarr, A., Andreasen, V., and Østergård, H. 2007. Dispersal distances for airborne spores based on deposition rates and stochastic modeling. Phytopathology 97:1325-1330.

A new modeling framework for particle dispersal is explored in the context of the particles being fungal spores dispersed within a field. The

concept is equivalent to the deposition rate for fungal spores. Special cases where parameter values for wind and gravitation lead to exponentially or polynomially decreasing densities are discussed, and formulas for one- and two-dimensional densities of deposited spores are given explicitly in terms of parameters for diffusion, wind, gravitation, and spore release height.
\end{abstract} model gives rise to both exponentially decreasing and polynomially decreasing two-dimensional densities of deposited fungal spores. We reformulate the model in terms of time to deposition, and show how this
Additional keywords: dispersal gradient, power law.
The dispersal of airborne fungal spores within a field has been subject to much discussion. A central point is whether spore dispersal distance from a single point source is best described by an exponentially decreasing density, corresponding to a focus around the source, or by a more heavy-tailed distribution in the form of a "power law" density, in the sense that the spore density is the reciprocal of a polynomial $(5,8,12,15)$. We shall refer to the last of these classes as "polynomially decreasing densities." There is no clear picture of when each of the two classes of densities describes fungal spore dispersal the best. Out of 124 studies of dispersal of dry airborne spores and pollen (8), the dispersal distance in 59 studies is better described by a polynomially decreasing density than by a standard exponential density, in the sense that the $r^{2}$ goodness-of-fit statistic reached a higher value for the polynomially decreasing density than for the exponentially decreasing density (7). For a fixed median travel distance, the difference between these two types of densities may be very hard to detect over small distances whereas, for longer distances, there will be a significant difference (Fig. 1) (15). For dry airborne spores, polynomially decreasing densities provide a better fit to dispersal distances than do exponentially decreasing densities when the fungal spore size is $<10 \mu \mathrm{m}$ (6). This result suggests that the observations have been made at a distance where exponentially decreasing and polynomially decreasing densities differ substantially (Fig. 1). The result also suggests that there is a critical spore size below which movement due to diffusion will be dominant compared with movement due to gravitation. However, the physical shape of the spore also may play a role.

The purpose of this article is to reformulate and extend a model which may lead to these different dispersal patterns (16). Consequently, we focus on distances of the order 10 to 100 times the median travel distance. These distances may be well within observable reach for the kind of dispersal considered here (7). In

Corresponding author: H. Østergård; E-mail address: hanne.oestergaard@risoe.dk

doi:10.1094/PHYTO-97-10-1325

(c) 2007 The American Phytopathological Society general, median travel distances may be difficult to observe because many spores deposit close to the source plant. However, in dispersal experiments, 20- $\mu \mathrm{m}$ droplets (simulating Erysiphe graminis spores) traveled a median distance of $<1 \mathrm{~m}$ when no wind effect was present (10). These distances are relevant for focal expansion within a field and, as such, are different from what often is called long-distance dispersal (3).

Until recently, no clear connection between the two types of densities for dispersal has been provided $(2,7)$. The two types have been suggested to relate to different kinds of mechanisms for fungal spore dispersal (19); for example, determined by weather conditions (2). Consequently, models have dealt with these mechanisms separately (4) or through a two-step procedure, depending on the distance from the source incorporating both mechanisms (20).

A theoretical background for understanding these two types of dispersal as essentially the same phenomenon recently has been provided, modeling the distribution of particles in general (16). In the context of fungal spores, the main difference to previous expositions is the emphasis on the combination of spore transport and deposition. Here, we elaborate on this approach, introduce diffusion coefficients into the formulas, and show how both exponentially and polynomially decreasing densities for dispersal are special cases of the same modeling framework. In addition, this framework provides a natural way of constructing two-dimensional densities that approximate spore dispersal.

In order to efficiently use diffusion modeling, the influence of environmental conditions on spore dispersal, such as wind speed, wind direction, and other factors that affect the transport of spores, must have stationarity properties in time such that spore transport, in both the vertical and horizontal directions, can be modeled by standard diffusion. In particular, we assume constant wind speed and wind direction, which is in contrast to averaging the wind effects over time. Because of the requirement of stationarity properties, long-distance dispersal in this context should be viewed as relative to median travel distance which, for some fungal spores, may be measured in meters. Thus, when constructing densities, we are not discussing dispersal on a conti- 
nental scale. In such situations, events such as escape to the upper atmospheric layers and varying environmental conditions makes the diffusion approximation problematic (3).

\section{THEORY AND APPROACHES}

Aerial spore dispersal from a point source may be divided into three processes: spore release, aerial transport, and deposition. Spore release often is not considered, and we shall only briefly state how we model it after having dealt with transport and deposition.

The spore transport model. Aerial transport usually is modeled by a two-dimensional diffusion process, neglecting transport in the vertical direction (17). In the simple diffusion model disregarding population growth, the spore density $n\left(t ; x_{1}, x_{2}\right)$ at point $\left(x_{1}, x_{2}\right)$ and time $t$ is given as the solution to the differential equation

$$
\frac{\partial n}{\partial t}=w_{1} \frac{\partial n}{\partial x_{1}}+w_{2} \frac{\partial n}{\partial x_{2}}+\frac{\sigma_{h}^{2}}{2}\left(\frac{\partial^{2} n}{\partial x_{1}^{2}}+\frac{\partial^{2} n}{\partial x_{2}^{2}}\right)
$$

The parameters are the horizontal drift vector $\left(w_{1}, w_{2}\right)$ due to a constant wind velocity, and the coefficient of constant horizontal diffusion $\sigma_{h}$. The latter measures the order of magnitude of
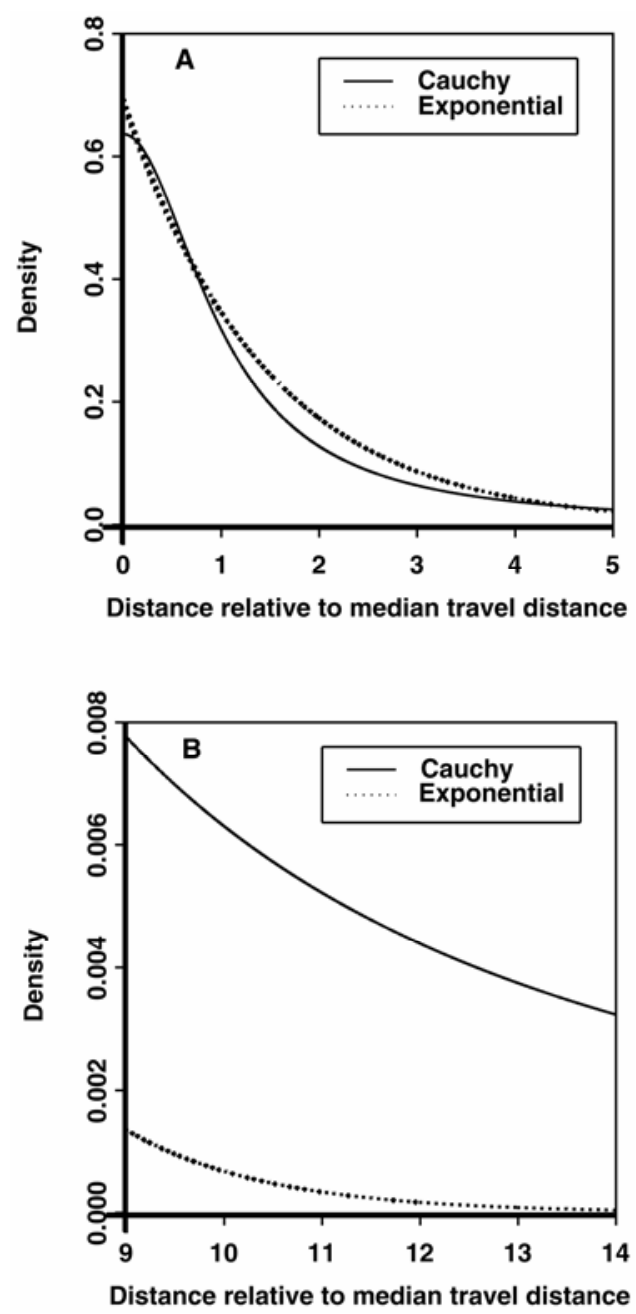

Fig. 1. Probability density of the standard Cauchy $f_{1}(x)=2 /\left[\pi\left(1+x^{2}\right)\right]$, with the horizontal axis being the median travel distance, and the probability density for the exponential distribution $f_{2}(x)=b^{-1} e^{-x / b}$, scaled with $b=1 / \log (2)$ to comply with the horizontal axis being the mean travel distance. A, Close to the source, the densities are close to being indistinguishable whereas $\mathbf{B}$, at 9 to 14 times the median travel distance away from the source, the difference between the densities is significant. random fluctuations of the spores, relative to the chosen time scale. If $N$ spores are released at origin, the solution to equation 1 leads to a time-dependent density of spores in the twodimensional plane (17):

$$
n\left(t ; x_{1}, x_{2}\right)=\frac{N}{2 \pi \sigma_{h}^{2} t} \exp \left(-\frac{1}{2 \sigma_{h}^{2} t}\left(\left(x_{1}-t w_{1}\right)^{2}+\left(x_{2}-t w_{2}\right)^{2}\right)\right)
$$

If $N=1$, we can consider the spore density equation 2 as a probability density.

The spore deposition model. To describe the deposition of spores, let the deposition rate $\phi(t)$ denote the expected proportion of the spores in the air that are deposited per time unit at time $t$. Assuming that the deposition rate $\phi$ is independent of the number of spores released $(N)$, it suffices to study the case of $N=1$. This allows us to express $\phi(t)$ in terms of the time to deposition $(\tau)$ (i.e., the time the spore travels from its point of release to its point of deposition). The time to deposition turns out to be a more natural concept in our formulation than the deposition rate. Using the definition of $\phi(t)$, we can approximate the probability that the travel time falls in the interval between $t$ and $t+\Delta t$, given that the travel time is longer than $t$ by the length of the time interval $\Delta t$ times the deposition rate. This can mathematically be formulated as $P$ (spore deposition takes place between $t$ and $t+\Delta t$ given that spore deposition has not taken place before $t) \approx \phi(t) \Delta t$.

Thus, $\phi$ acts as the intensity for $\tau$, which induces a direct relationship between $\phi$ and the distribution of $\tau$. If we assume that $\phi$ is continuous, and denote the probability density for the time to deposition $\tau$ by $f$, then the relationship between $\phi$ and the distribution of $\tau$ is as follows:

$$
P(\tau>t)=e^{-\int^{t} \phi(s) d s}
$$

and

$$
\phi(t)=f(t) /\left(1-\int_{0}^{t} f(s) d s\right)
$$

so that any choice of a deposition rate $\phi$ specifies the distribution of $\tau$ and vice versa. In this sense, the time to deposition offers an alternative formulation of the concept of deposition rate. Our approach to spore deposition (16) is most conveniently described through the time to deposition, because it naturally links the concept of vertical diffusion to the notion of deposition rates through equations 3 and 4 and allows us to compare models where the spore deposition is specified through a deposition rate only.

The dispersal model. A new way of modeling the dispersal process has been considered, which may be described through the time to deposition (16). The diffusion model that corresponds to equation 1 is extended to three dimensions, and the corresponding differential equation is solved subject to two initial conditions: (i) the spores are released at a positive height $a$ and (ii) the diffusion stops when the particle is being deposited, at the time-point which we define as the first time the particle reaches a certain height $b$ (e.g., ground level), with $b<a$.

The term "release height" is not to be taken literally. In this context, release height is used as a metaphor to describe the outcome of the complicated turbulent diffusion processes in the crop boundary layer that brings the spores from the actual release point on the plant to the region where diffusion describes spore movement $(1,2)$. The corresponding deposition rate will then be nonconstant over time. These assumptions applied to the model (equation 1) lead to a resulting two-dimensional density $n\left(x_{1}, x_{2}\right)$ of deposited spores, which equals a weighted average of densities $n\left(t ; x_{1}, x_{2}\right)$ of the form given in equation 2 where the weights are given by the probability density of the time to deposition.

The three-dimensional extension of the model (equation 1) determines the time-to-deposition $\tau$. Using standard diffusion techniques (14), it is possible to calculate an explicit expression 
for the corresponding probability density $f(t)$ for $\tau$ as follows. Letting $\gamma$ represent a constant downward drift velocity in the three-dimensional model due to gravitation, denoting the vertical diffusion coefficient by $\sigma_{v}$ and taking $\tilde{a}=(a-b) / \sigma_{v}$ and $\tilde{\gamma}=\gamma / \sigma_{v}$, we find that

$$
f(t)=\frac{\tilde{a}}{\sqrt{2 \pi}} t^{-3 / 2} \exp \left(-\frac{(\tilde{a}-\tilde{\gamma} t)^{2}}{2 t}\right), \quad t>0
$$

Note that if we chose a different re-parametrization for $\gamma$ : $\tilde{\gamma}=\sigma_{v}^{-2}(a-b) \gamma$, the parameter $\tilde{a}^{2}$ will act as a scaling parameter, scaling the axis to which the probability density applies. This also holds true if the described particles are so light that no vertical drift applies (i.e., $\gamma=0$ ).

In analogy with the release height parameter $a$, a word of caution should be added to the assumption $\gamma=0$. The parameter $\gamma$ is related to a probability density for fungal spores and, although it has the interpretation of gravity, it is not directly translatable as such. The parameter summarizes the forces that act in connection with the downward pull, which also involves the shape of the spore and atmospheric effects. As such, the context of the assumption $\gamma=0$ is the probabilistic model, where the effect of gravity is assumed to be inferior compared with other forces at work. Thus, when putting the hypothesis $\gamma=0$ forward, we claim that the effect of gravity is negligible. This interpretation is consistent with findings in (6), where there appears to be a limit of $\approx 10 \mu \mathrm{m}$ in particle diameter for when exponentially decreasing densities no longer provide the best fit when compared with polynomially decreasing densities.

In conclusion, the time it takes for a spore to travel to the point of deposition will follow the probability distribution specified by the probability density given by equation 5 . From this, we can calculate the deposition rate $\phi(t)$ from the model given by equation 1 , using the relationship with $\tau$ given by equation 4 .

The two-dimensional probability density for spore dispersal. From the dispersal model, it is possible to calculate a general expression for the spatial probability density of our single deposited spore. The spore densities (equation 2) averaged with respect to the time-to-deposition probability density (equation 5) takes the form

$$
\begin{aligned}
& g\left(x_{1}, x_{2}\right)=\frac{\tilde{a}}{\sigma_{h}^{2}(2 \pi)^{3 / 2}} \exp \left(\tilde{x}_{1} \tilde{w}_{1}+\tilde{x}_{2} \tilde{w}_{2}+\tilde{\gamma} \tilde{a}\right) \\
& \times \int_{0}^{\infty} t^{-5 / 2} \exp \left\{-\frac{1}{2}\left(\left(\tilde{a}^{2}+\tilde{x}_{1}^{2}+\tilde{x}_{2}^{2}\right) t^{-1}+\left(\tilde{\gamma}^{2}+\tilde{w}_{1}^{2}+\tilde{w}_{2}^{2}\right) t\right)\right\} d t
\end{aligned}
$$

where $\tilde{a}=(a-b) / \sigma_{v}, \quad \tilde{\gamma}=\gamma / \sigma_{v}, \quad \tilde{x}_{i}=x_{i} / \sigma_{h}, \tilde{w}_{i}=w_{i} / \sigma_{h}, i=1,2$. We shall return to possible simplifications of equation 6 in the next section.

When many spores instead of one are released from the source (in the following, we thus assume that $N>1$ ), they will be deposited over time according to the spore density $N \times f(t)$ from equation 5 . We assume that spores are released over a period where the environmental parameters are constant. Thus, the $N$ spores are not necessarily released at the same instant. However, this will not affect the number of spores, because we choose to study dispersal "after all spores have been deposited." Spores that spend $t$ time units traveling to their destination will distribute themselves in space according to the probability density $n\left(t ; x_{1}, x_{2}\right)$ from the model (equation 2). Therefore, the probability density in equation 6 (multiplied by $N$ ) yields a density describing the spatial distribution of the spores that are dispersed from the given source.

Dispersal over several generations. A further development of the model with the probability density in equation 6 is to consider the tail of the densities, after spores have been deposited for a fixed number of generations. The distance to the original point of release, in two dimensions or along any line transect, then may be derived as a convolution of the densities for one dispersal event. This changes the shape of the probability density: if a probability density is exponentially decreasing along a given line transect as distance from the source increases, it may be approximated by a straight line on a logarithmic scale at large distances from the original point of release. The slope of this line, the dispersal gradient (6), will not be sensitive to convolutions, although convergence to this straight line will happen further away from the original point of release.

As distance from the original point of release increases, polynomial type densities can be approximated by a straight line on a double-logarithmic scale. We shall refer to the slope of this line as a "dispersal gradient" because the gradient characterises the decrease in spore concentration with distance and, thus, the dispersal. Our concept of dispersal gradient is consistent with the methods suggested by McCartney and Fitt (11, equations 7.10 and 7.11). In this case, it also can be shown that the dispersal gradient remains unchanged, although the shape of the probability density may be altered.

A special case of an exponentially decreasing probability density is the standard exponential probability density with mean $\beta$ (5). This density has a dispersal gradient of $1 / \beta$, and corresponds to a straight line on a logarithmic scale. By convolution, the probability density will be of the form of a polynomial times a standard exponential function, thus not changing the dispersal gradient, but the convergence to the straight line does not happen before the polynomial is insignificant in comparison with the exponential function. With each convolution, this will happen further away from the original point of release.

\section{RESULTS}

In a number of situations, the probability density (equation 6) can be simplified significantly. We shall consider an expansion of three simplifications presented by Stockmarr (16), where we elaborate on the effect of the parameters.

Case 1: $\gamma=0$ and $\boldsymbol{w}=\mathbf{0}$. In the simple case where the effect of both gravitation and wind are ignorable compared with the effect of diffusion, the probability density in equation 6 simplifies to

$$
g\left(x_{1}, x_{2}\right)=\frac{\tilde{a}}{2 \pi \sigma_{h}^{2}\left(\tilde{a}^{2}+\tilde{x}_{1}^{2}+\tilde{x}_{2}^{2}\right)^{3 / 2}}
$$

with the same notation. Consequently, the distance $r$ between the spore source and the point of deposition has a probability density given by

$$
g_{\text {dist }}(r)=\frac{\beta r}{\left(\beta^{2}+r^{2}\right)^{3 / 2}}
$$

where $\beta=(a-b) \sigma_{h} / \sigma_{v}$. This corresponds to the square of the distance between the point of release and the point of deposition being $F(2,1)$-distributed with scale parameter $\beta^{2}$.

The density of spores deposited along a line also may be derived from equation 6 . This type of density corresponds to the van den Bosch et al. (17) concept of marginal probability density. The density is given by

$$
g_{\text {marg }}(x)=\frac{\beta}{\pi\left(\beta^{2}+x^{2}\right)}
$$

where $x$ denotes the position of the spore source along the line, and we recognize the probability density for the standard Cauchy distribution, scaled by $\beta$.

The Cauchy probability density has a dispersal gradient of -2 . When considering dispersal over a several generations along a line according to equation 8 , the dispersal gradient will remain unchanged, because convolution of Cauchy distributions does not affect the dispersal gradient. 
Case 2: $\gamma=0$ and $\boldsymbol{w} \neq 0$. If the effect of gravitation can be ignored, but a nonnegligible (constant) wind effect is present, the density of spores along a line transect in the wind direction is described through the probability density

$$
f_{1}(x)=\int_{0}^{\infty} \frac{1}{\sqrt{2 \pi \sigma_{h}^{2} t}} \exp \left(-\frac{1}{2 t}(\tilde{x}-\tilde{w} t)^{2}\right) \times \frac{\tilde{a}}{\sqrt{2 \pi}} t^{-3 / 2} \exp \left(-\frac{\tilde{a}^{2}}{2 t}\right) d t
$$

where the " $\sim$ "-quantities are as in equation 6 . The tail of this probability density is approximated (16) by

$$
f_{1}(x) \sim \sqrt{\frac{2 \tilde{w} \tilde{a}^{2}}{\pi^{3} \sigma_{h}^{2}}}\left(\tilde{a}^{2}+\tilde{x}^{2}\right)^{-3 / 4} \exp \left(-\tilde{w}\left(\sqrt{\tilde{a}^{2}+\tilde{x}^{2}}-\tilde{x}\right) \text { as } x \rightarrow \infty\right.
$$

It can be deduced from equation 10 that the probability density (equation 9) is asymptotically proportional to $x^{-3 / 2}$, with the coefficient of proportionality being

$$
\sqrt{2 \sigma_{h} \tilde{w} \tilde{a}^{2} \pi^{-3}}=\sqrt{2 w} \pi^{-3 / 2}(a-b) / \sigma_{v}
$$

Thus, the probability density in the wind direction has a tail that is even heavier than the Cauchy probability density. However, this is the only direction in which the density of deposited spores is not exponentially decreasing. The two-dimensional probability density is shown in Figure 2.

When describing the spatial distribution of dispersed spores, the two-dimensional probability density (equation 6) should be considered rather than simplifications, because the probability density is not rotationally symmetric, meaning that the densities along line transects depends on the angle to the wind direction. Because of the wind effect, the probability density in equation 9 is a density with infinite mean along the line transect given by the wind direction, indicating that a significant number of the spores will travel far away from the source. This is not the case along line transects in all other directions.

When considering several generations, there does not seem to be a simple expression for the convolution of the probability density in equation 9. However, equation 10 shows that the density (equation 9) is asymptotically a polynomial-type probability density and, because convolutions do not change the dispersal gradient for polynomial-type densities, this applies to equation 9 as well.

Case 3: $\gamma>0$. Suppose that the effect of gravitation cannot be ignored (i.e., we cannot assume that $\gamma=0$ ). In this case, an analytical expression of equation 6 can be derived in a form that shows that the two-dimensional probability density will decrease exponentially in all directions (16). The dispersal gradient is equal to $-\gamma$ in all directions in the absence of wind and, otherwise, the probability density is steepest in the opposite direction of the wind, declining as the angle to the wind direction decrease. How-

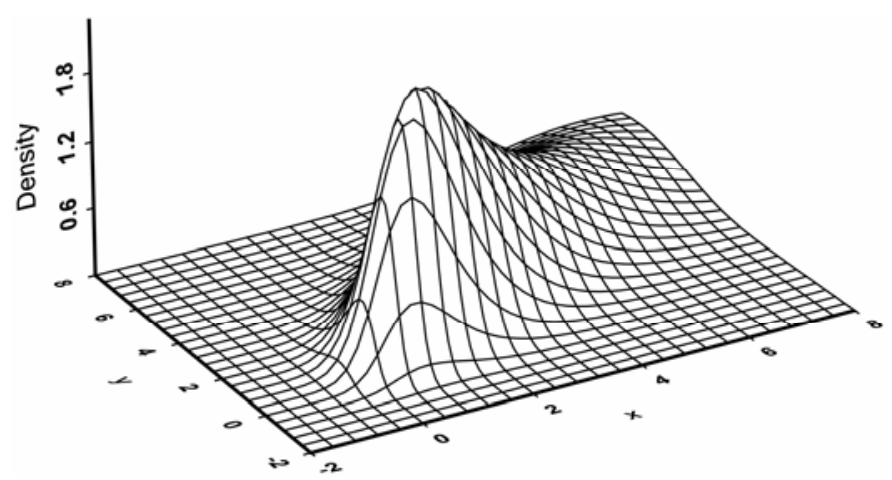

Fig. 2. Two-dimensional density in the case of no gravitational effect, but presence of a horizontal, constant wind effect at the $45^{\circ}$ angle. The density is exponentially decreasing in all directions except the line segment in the wind direction, where the decrease is according to a polynomially decreasing density. ever, even in the wind direction, the probability density decreases exponentially, regardless of the size of the wind effect (16).

When considering several generations, the convolutions will all have exponentially decreasing densities, as explained in the section on dispersal over several generations.

Some statistical aspects. The parameters involved in the spore dispersal density (equation 6) are $\sigma_{h}, \sigma_{v}, \widetilde{w}, \tilde{\gamma}$, and $\tilde{a}$. The diffusion coefficients, wind parameters, and gravitation can be estimated based on dispersal properties of the fungal spores such as weight and shape, whereas the scaled distance $\tilde{a}=(a-b) / \sigma_{v}$ between release and deposition height will require further information about the fungus. We emphasize that the diffusion coefficients are relative to the chosen time and space scale. For example, in the absence of wind, a spore will travel, on average, $\sqrt{2 \pi} \sigma_{h}$ horizontally away from the source per time unit, assuming that it is not deposited. Therefore, choice of scales and diffusion coefficients are connected. If information on some parameters is not sufficiently detailed to allow for a quantitative estimation, these parameters can be considered as free parameters that are to be estimated from data.

The model framework covers a wider class of models than those with a constant wind effect. If the spore release period can be divided into periods each having constant wind effects, although in different directions, a set of conditional models for each period can be combined into an overall model. This procedure is different from applying the model in equation 6 with an average wind effect. The procedure has been applied on Puccinia graminis spore data (K. D. Bjerre, unpublished data), where models as described above with two wind directions provided consistently better fits than models with average wind parameters.

\section{DISCUSSION}

By including an explicit description of the spore deposition process, we show how diffusion models can lead to types of tails other than exponentially decreasing density. We will discuss special features of the diffusion models below.

Most studies do not specify explicitly how deposition takes place and common approaches result in spore densities decreasing exponentially with the distance from the source. As an example, assuming that the deposition rate $\phi(t)$ is constant over time (17), an exponentially decreasing density of deposited spores along any line transect is obtained when combined with the probability density in equation 2 (18). Thus, with a constant deposition rate, the model in equation 1 will not predict polynomially decreasing densities for deposited spores. Another approach is to assume that spores dispersed according to the model in equation 1 are being deposited after a fixed time to deposition $T$. The resulting twodimensional spore density will be of the form $n\left(T i, x_{1}, x_{2}\right)$ (equation 2), corresponding to the Gaussian Plume Model (7); again, an exponentially decreasing and not a polynomially decreasing density of deposited spores is obtained.

The difference between the constant deposition rate and the approach in this article is illustrated by exemplified flights, where a dispersal process is simulated from equation 1 with a $45^{\circ}$ wind effect of size 0.5 and stopped at the time to deposition (Fig. 3). The time to deposition is simulated twice: first, according to a constant deposition rate (Fig. 3A, 1-A3) where the time to deposition is found through equation 3 ; and second, according to the deposition rate derived from the hitting time of zero height for a vertical Brownian motion started at unit height (Fig. 3B, 1-B3), corresponding to the probability density in equation 5 . In the boxes $\mathrm{A} 1$ and $\mathrm{B} 1$, the constant deposition rate gives a longer time to deposition than the vertical diffusion, 8.43 versus 2.30 , but the distance traveled from the point of release is of the same order of magnitude; B1 is recognized as approximately one-third of the whole flight pictured in A1. Conversely, boxes A2 and B2 show an exemplified flight where the vertical diffusion gives a longer 
time to deposition than the constant deposition rate, 606.25 versus 2.14. Here, the distance traveled from the point of release is of different magnitudes, and A2 cannot be recognized as the first part of B2 without considerable magnification, despite the two models having the same median. In essence, the phenomenon illustrated is that, although the two deposition rates have identical medians, the mean time to deposition in the former case is 3.16 whereas the mean time to deposition is infinite in the latter. This is further illustrated in boxes A3 and B3, which each shows 10 simulated dispersals, including the special cases above (note that the end point for the dispersal in boxes A1, A2, B1, and B2 is not necessarily the point furthest to the right). In the former case, a regular point pattern around the $45^{\circ}$ regression line is shown whereas, in the latter, a diffuse point pattern is shown with a number of spores clustered relatively close to the point of release while other spores travel a long distance.

A technique similar to that used to obtain equation 6 has been used to construct a pollination time from the same principles as our time to deposition (9). However, insisting on a strictly negative gravitation, they did not reach the same thick-tailed distributions as we will demonstrate below.

When applying our model to dispersal over multiple generations, the shape of the spore density can be determined by convo-

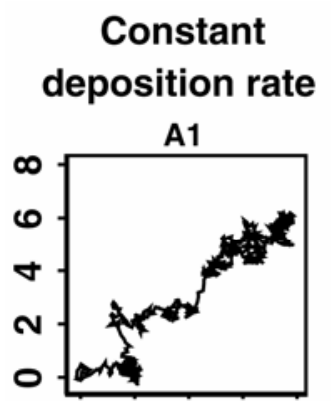

\section{Vertical diffusion deposition rate}
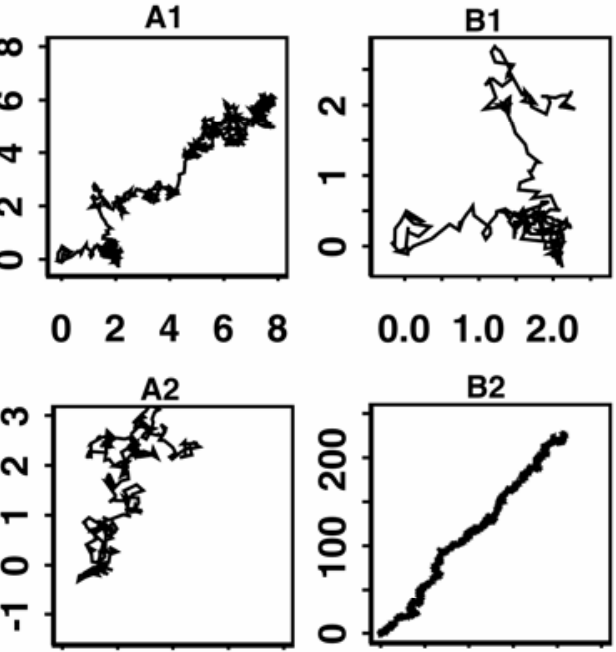

0.01 .02 .0
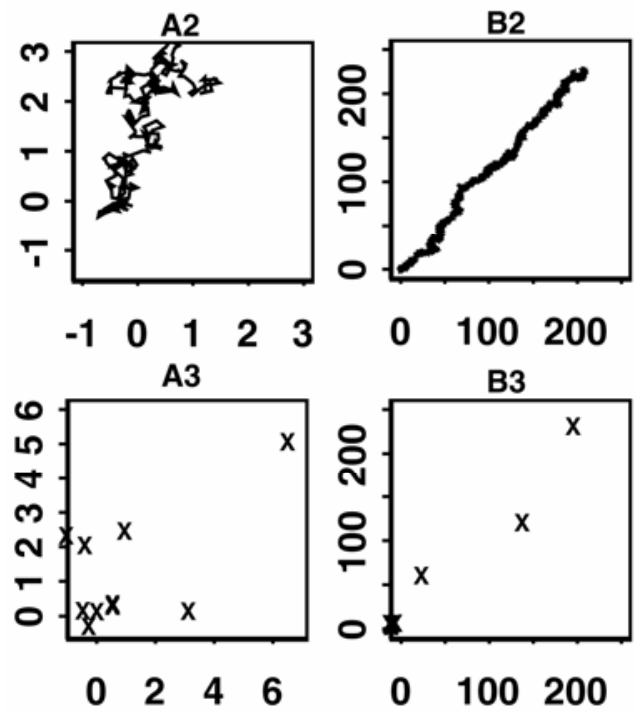

Fig. 3. Exemplified flight patterns on an arbitrary scale for fungal spores with a drift of 0.5 in the $45^{\circ}$ direction: Dispersal is simulated from equation 1 and stopped at the time to deposition, which is simulated twice: $\mathbf{A}$, constant deposition rate and $\mathbf{B}$, deposition rate determined by the hitting time for height 0 for a Brownian motion started at unit height, corresponding to the probability density in equation 5 . In both situations, the median time to deposition is 2.19 time units. The two upper pairs compare a spore flight where the time to deposition attains the highest value for $\mathrm{A}$ in the pair (A1, B1) and B in the pair (A2, B2), respectively. The lower pair (A3, B3) shows simulated deposition points of 10 spores under the two simulations, including those from the upper pairs. lutions. However, this approach requires that the number of generations is fixed and well defined. In determining whether this is the case, the latent and infectious periods of the fungus are important factors to consider. Unless the latent period is significantly longer than the infectious period plus the time to deposition, spores of one generation will be released before all spores of the previous generation are deposited. The number of generations is then no longer well defined, and the validity of the convolution method is unclear. Unfortunately, we are unable to determine the validity of our approach in relation to another simulation study with overlapping generations (15) because the article does not include a probability density for the distance from distributed spores to the initial spore source at the end of the simulation.

Several authors have dealt with atmospheric models for spore dispersal which operate with three-dimensional diffusion models (2). The main difference to what is presented here is that the diffusion coefficient in the third coordinate for these models is nonconstant; the reason being that, close to the ground, the diffusion will decrease toward 0 . Although providing more detailed models, the mathematical complexity of this approach limits the possibilities of deriving general results. A model for deposited spores along the line transect corresponding to the wind direction has been derived based on a nonconstant vertical diffusion coefficient (13) and, for specific choices of the vertical diffusion, a probability density along this transect is computed. However, these authors ignore the horizontal diffusion; thus, essentially, they describe the time to deposition. The present approach is based on extending the standard horizontal diffusion model (equation 1), and this makes our results noncomparable.

The approach taken in this article results in much simpler formulas than would have been the case if the vertical diffusion had been allowed to vary. Although we acknowledge that there may be situations where the improvement in fit justifies the complexity of allowing for nonconstant vertical diffusion, it is our opinion that this does not apply to spore dispersal in the form considered here, because a vertical diffusion increasing with height will tend to make the effects on the asymptotic behavior of the dispersal densities more pronounced rather than blurring them (16). Thus, we note that such a modification will not change the basic message of the extension to three dimensions: that the spore densities derived from equation 1 fall into the two categories of exponentially decreasing and polynomially decreasing densities, respectively, and that the appropriate category is determined by the actual influence of wind and gravitation.

In conclusion, fungal spores dispersed widely relative to the median travel distance can be modeled by diffusion, and it is possible to rely on the advantages of diffusion modeling even though observed dispersals do not fit into constant wave velocity models. In this way, theoretical epidemiology can take advantage of the large mathematical toolbox which diffusion modeling provides.

\section{ACKNOWLEDGMENTS}

This research was funded by the Danish Environmental Research Programme. We thank K. D. Bjerre for stimulating discussions related to applications to observed data of the model, and M. W. Shaw for his part in discussions on interpretation of the model and for his useful comments to earlier versions of the manuscript.

\section{LITERATURE CITED}

1. Aylor, D. E. 1990. The role of intermittent wind in the dispersal of fungal spores. Annu. Rev. Phytopathol. 28:73-92.

2. Aylor, D. E. 1999. Biophysical scaling and the passive dispersal of fungal spores: Relationship to integrated pest management strategies. Agric. For. Meteorol. 97:275-292.

3. Aylor, D. E. 2003. Spread of plant disease on a continental scale: role of aerial dispersal of pathogens. Ecology 84:1989-1997. 
4. Bullock, J. M., and Clarke, R. T. 2000. Long distance seed dispersal by wind: Measuring and modelling the tail of the curve. Oecologica 124:506521.

5. Ferrandino, F. J. 1996 Length scale of disease spread: fact or artifact of experimental geometry. Phytopathology 86:806-811.

6. Fitt, B. D. C., Gregory, P. H., and Todd, A. D., McCartney, H. A., and McDonald, O. C. 1987. Spore dispersal and plant disease gradients: A comparison between two empirical models. J. Phytopathol. 118:227-242.

7. Fitt, B. D. C., and McCartney, H. A. 1986. Spore dispersal in relation to epidemic models. In: Plant Disease Epidemiology, vol. 1: Population Dynamics and Management. K. J. Leonard and W. E. Fry, eds. Macmillan Publishing Company, New York.

8. Gregory, P. H. 1968. Interpreting plant disease dispersal gradients. Annu. Rev. Phytopathol. 6:189-212.

9. Klein, E. K., Lavigne, C., Foueillassar, X., Gouyon, P.-H., and Laredo, C. 2003. Corn pollen dispersal: Quasi-mechanistic models and field experiments. Ecol. Monogr. 73:131-150.

10. McCartney, H. A., and Bainbridge, A. 1984. Deposition gradients near to a point source in a barley crop. Phytopathol. Z. 109:216-236.

11. McCartney, H. A., and Fitt, B. D. L. 1998. Dispersal of foliar fungal plant pathogens: Mechanisms, gradients and spatial patterns. Pages 138-160 in: The Epidemiology of Plant Diseases. D. G Jones, ed. Kluwer Publishers,
Dordrecht, The Netherlands.

12. Mundt, C. C., and Leonard, K. J. 1985. A modification of Gregory's model for describing plant disease gradients. Phytopathology 75:930-935.

13. Okubo, A., and Levin, S. 1989. A theoretical framework for data analysis of wind dispersal of seeds and pollen. Ecology 70:329-338.

14. Rogers, L. C. G., and Williams, D. 1994: Diffusions, Markov Processes and Martingales. Volume I: Foundations. Second Edition. Wiley, Chichester, UK.

15. Shaw, M. W. 1995. Simulation of population expansion and spatial pattern when individual dispersal distributions do not decline exponentially with distance. Proc. R. Soc. Lond. B 259:243-248.

16. Stockmarr, A. 2002. The distribution of particles in the plane dispersed by a simple 3-dimensional diffusion process. J. Math. Biol. 45:462-469.

17. van den Bosch, F., Metz, J. A. J., and Diekmann, O. 1990. The velocity of spatial population expansion. J. Math. Biol. 28:529-565.

18. Williamson, E. J. 1961. The distribution of larvae of randomly moving insects. Aust. J. Biol. Sci. 14:598-604.

19. Zawolek, M. W. 1993. Shaping a focus: Wind and stochasticity. Neth. J. Plant Pathol. 99:241-255.

20. Zawolek, M. W., and Zadoks, J. C. 1992. Studies in focus development: An optimum for the dual dispersal of plant pathogens. Phytopathology 82:1288-1297. 